\title{
LENGUA Y ARCHIVO: GLOTOPOLÍTICA E HISTORIOGRAFÍA LINGÜÍSTICA DESDE LAS PÁGINAS DE ANCLAJES
}

\author{
Juan Antonio Ennis \\ Instituto de Humanidades y Ciencias Sociales, IdHICS \\ Universidad Nacional de La Plata \\ Consejo Nacional de Investigaciones Científicas y Técnicas, CONICET \\ Argentina \\ juanennis@conicet.gov.ar \\ ORCID: 0000-0002-7070-464X
}

Fecha de recepción: 29/06/202I | Fecha de aceptación: I2/07/202 I

\begin{abstract}
Resumen:A mediados del siglo XX emergen perspectivas que estudian el lenguaje en la sociedad y logran su apogeo en los estudios sobre ideologías de las lenguas a fines del mismo siglo y los más recientes desarrollos de la glotopolítica y la historiografía lingüística. Unas y otras perspectivas comparten la certeza de que la lengua en la modernidad es un artefacto discursivo cuya historia y construcción social es eminentemente política. Se reseñan los aportes de estas perspectivas teórico-críticas y se los relaciona con algunas investigaciones publicadas en la revista Anclajes.
\end{abstract}

Palabras clave: lengua; archivo; glotopolítica; historiografía lingüística; Argentina.

\section{Language and archive: glotopolitics and linguistic historiography from the pages of Anclajes}

\begin{abstract}
Studies about the relationship between language in society emerged in the mid-20th century, and reached a peak at the end of the century with analyses on language ideologies and the most recent developments in glotopolitics and linguistic historiography. Both perspectives share a common framework: that language in modernity is a discursive artifact whose history and social construction is eminently political. The contributions of these theoretical-critical perspectives are reviewed and related to some research published in the journal Anclajes.
\end{abstract}

Keywords: language; archive; glotopolitics; linguistic historiography; Argentina. 


\section{Linguagem e arquivo: glotopolitica e historiografia linguística das páginas de Anclajes}

Resumo: Em meados do século $X X$, surgiram perspectivas que estudava a linguagem na sociedade e alcançava seu apogeu nos estudos sobre ideologias das línguas no final do mesmo século e nos mais recentes desdobramentos da glotopolítica e da historiografia linguística.Ambas as perspectivas compartilham a certeza de que a linguagem na modernidade é um artefato discursivo cuja história e construção social são eminentemente políticas.As contribuições dessas perspectivas teórico-críticas são revisadas e relacionadas a algumas pesquisas publicadas na revista Anclajes.

Palavras chave: língua;Arquivo; glotopolítica; historiografia linguística;Argentina.

L

as ciencias del lenguaje se establecen y afianzan, en sus distintas modulaciones hegemónicas a lo largo de los siglos XIX y XX, afirmando por un lado la esencial importancia de la lengua para la conformación de la vida social y la comunidad política, y procurando al mismo tiempo desprenderla de ellas, para dar con un objeto susceptible de caber en la sistematicidad de un modelo científico où tout se tient. La crítica a este modelo descarnado de la lengua como objeto de estudio (lo que Roy Harris llamó hace cuarenta años "el mito de la lengua") y de la consecuente división fundante entre lingüística interna y lingüística externa encuentra diversas manifestaciones en el tiempo, pero comienza a consolidarse sobre todo en la segunda mitad del siglo XX con la emergencia de perspectivas como la sociolingüística, entre otras, abocadas a indagar el lenguaje en la sociedad, y no a pesar de ella. Este desprendimiento de lengua y sociedad tenía también como consecuencia un desprendimiento de la lengua y la historia, contra el que argumentaba Tony Crowley en su Language in History, releyendo a Mijail Bajtín. El Raymond Williams de Marxismo y literatura ya lo había hecho veinte años antes, recuperando a Valentín Volóshinov. En ese segundo capítulo dedicado a la lengua, Williams, con su recorrido desde Johann T. Herder hasta Noam Chomsky, demostraba ya cómo a esta altura, para entender el lenguaje en la sociedad, había que dar cuenta de la historia de los saberes y discursos autorizados para dar cuenta de él. Algo similar a lo que había hecho Michel Foucault diez años antes, al postular que de algún modo ese discurso relegado a "los márgenes de nuestra consciencia histórica”, que habría fundado una disciplina "un tanto lateral y esotérica” (específicamente la gramática histórico-comparativa de Franz Bopp, Jacob Grimm, Rasmus Rask y August W. von Schlegel), habría modificado "todo el modo de ser del lenguaje" (Las palabras 275). Esta conciliación del archivo de la disciplina y la carnadura política y social del objeto encontraría expresión en los ańos 1990 en la conformación de un campo dedicado a las ideologías lingüísticas, y especialmente en los volúmenes editados por Kathryn Woolard, Bambi Schieffelin y Paul Kroskrity en 1998, 
Kroskrity en el 2000 y Jan Blommaert en 1999. Allí este último establecía la relevancia del estudio de los debates sobre la lengua por el hecho de que eran estos los que podían motorizar sus procesos de cambio: "language is being changed by debates" (Blommaert 435). Como puede comprobarse en la amplia variedad de publicaciones (entre las que pueden destacarse los volúmenes de Richard Bauman y Charles Briggs de 2003, los posteriores de Joseph Errington o Tuska Benes en 2008 y el más reciente de Monica Heller y Bonnie McElhinny en 2017, entre otros), la historia de los saberes especializados y dispositivos de conocimiento, sistematización y eventual regimentación sobre el lenguaje resulta inescindible de la historia de la expansión colonial moderna de occidente también conocida como capitalismo. Dicho de otro modo, el espacio de investigación en torno al consenso de que lo que conocemos como lengua en la modernidad es un artefacto discursivo cuya historia y construcción social es eminentemente política (Del Valle Historia politica 22), no ha cesado desde entonces de expandirse.

Este tipo de desarrollos tienen en nuestro espacio sus formas específicas. Estas no dejan de vincularse con aquellos, pero al hacerlo, imprimen a la cuestión un giro propio, específico, relacionado no solamente con las características del campo en el que se desenvuelven, sino sobre todo con la impronta que el lugar de enunciación brinda a la construcción epistemológica.

Es el caso, por ejemplo, de la glotopolítica.

En 1986, Louis Guespin y Jean-Baptiste Marcellesi publicaban en la revista Langages un artículo-manifiesto llamado "Pour la glottopolitique", destinado a intervenir en las prácticas de la lingüística en su relación con la planificación de políticas del lenguaje, dicho de otro modo al desarrollo de una conciencia más clara y crítica del rol político de las y los profesionales de los estudios lingüísticos, sobre todo cuando su investigación funge como input para políticas estatales y formación de opinión que inciden en la vida de la comunidad hablante. Al filo del siglo, Elvira Arnoux recupera esta intervención, con explícita conciencia de los límites que la misma encontrara en su lugar de origen, para imprimirle así un giro desde la especificidad latinoamericana, como campo de trabajo y espacio de enunciación, que permitiría asimismo una consolidación más segura del término como demarcador de un espacio disciplinar, allí donde no solamente "la globalización muestra un rostro diferente" sino que además "América Latina constituye un espacio donde todavía pesan las tareas políticas no realizadas" (Arnoux "La glotopolítica"). El giro que proponía este artículo fundador, como lo reconoce un repaso reciente sobre la consolidación de la corriente de estudios reunida bajo el nombre de glotopolítica, implicaba asimismo agregar a la mirada sobre el presente de aquellos "hechos de lenguaje donde la acción de la sociedad reviste la forma de lo político" (Guespin y Marcellesi), otro ojo atento a la historia de los saberes especializados sobre el lenguaje: "Arnoux propose déjà une extension de leur approche en invitant à repenser radicalement les sciences du langage, en mettant au cœur de cette entreprise une focale sur l'histoire des idées linguistiques comme foncièrement ancrée dans une analyse des conditions 
politiques et des effets de domination que ces idées produisent, en questionnant les conditions matérielles de production des discours sur la langue, de même qu'en considérant que l'étude du langage s'accompagne d'un engagement politique assumé" (Arnoux, del Valle y Duchêne 2). Desde ese artículo hasta el presente, la glotopolítica se ha afirmado como corriente de pensamiento y espacio de producción, debate e intercambio, afianzada en los sucesivos congresos de la especialidad desde 2015, y en la aparición desde 2017 del Anuario de Glotopolitica, y en la aparición primero en inglés por Cambridge University Press (2013), luego en español por Aluvión (2015) de la Historia política del español, seguida recientemente por su crestomatía en el Autorretrato de un idioma. Este afianzamiento fue propiciado especialmente por la confluencia bajo el paraguas conceptual del término de los equipos coordinados por Arnoux en Buenos Aires con el de José del Valle -cuyos trabajos, sobre todo La batalla del idioma y La lengua ¿patria común? habían contribuido decisivamente a abrir camino a este tipo de investigaciones- en la City University of New York, que rápidamente fue integrando a numerosos equipos de trabajo y personal académico de distintas partes de Europa y América.

Al mismo tiempo, desde los años 70 del pasado siglo asistimos a la progresiva consolidación internacional de un espacio disciplinar específico dedicado al estudio de la historia de las ciencias del lenguaje, sobre todo a partir del establecimiento de la revista dirigida por uno de sus representantes más prolíficos y notorios, E.F.K. Koerner, Historiographia Lingüistica y las distintas publicaciones, sociedades y reuniones periódicas que fueron marcando la expansión de la disciplina hasta el día de hoy. Este desarrollo se cristaliza aquí cuando, con sede en el mismo Instituto de Lingüística de la Universidad de Buenos Aires desde el que se desarrolla la tarea de Arnoux y equipo, se comienza a publicar en 2009 la Revista Argentina de Historiografía Lingüistica, dirigida por Guillermo Toscano y García, y pensada asimismo como un espacio destinado a la difusión de las investigaciones sobre la historia de las ideas lingüísticas en América Latina (Toscano y García "Presentación"), que se sostiene hasta la fecha con periodicidad semestral y en permanente diálogo con los demás enfoques sobre el mismo objeto. Si bien la historiografía lingüística reviste su especificidad disciplinar, el deslinde teórico-metodológico que esto implica no excluye las inevitables y provechosas formas diversas del intercambio, la convivencia y muchas veces imbricación con las demás formas de acceso al mismo archivo de los saberes especializados sobre la lengua y sus procesos de desarrollo, afianzamiento y legitimación. De hecho, el primer número de la revista contiene en la reseña que José del Valle hace del imprescindible volumen de Arnoux sobre la lengua en la formación del Estadonación en Chile (Arnoux Los discursos de la nación), un espacio para destacar los rasgos específicos del enfoque glotopolítico allí asumido, en el cual del Valle anticipa los rasgos del proyecto común de elaboración teórica que cristalizarían en trabajos como el publicado al año siguiente en Spanish in Context (Arnoux y del Valle "Las representaciones"). 
En suma, un rápido vistazo sobre los estudios de esta índole en el país permite comprobar que hacia el final de la primera década de este siglo que vivimos se hace evidente una mayor proliferación y progresiva consolidación de este tipo de trabajos y las distintas perspectivas teórico-metodológicas para su abordaje, síntoma también del "giro archivístico" propio de los tiempos (Sánchez-Macedo 2020; Tello 2018: 11, e. o.). Dicho de otro modo, si bien los estudios en el área no carecían de antecedentes, el volumen y la profundidad de la investigación necesaria para consolidar el espacio y los equipos de trabajo necesarios para dar cuenta del archivo de los saberes y discursos legos y especializados que intervienen en la configuración discursiva de la(s) lengua(s) como acontecer político, en el país y la región principalmente, se hace por entonces más notable.

$\mathrm{Al}$ mismo tiempo, la materia comienza a recibir un renovado interés de parte de los estudios literarios, conformándose un espacio disciplinarmente plural, abierto a una diversidad de enfoques que encontrarían en las páginas de Anclajes un espacio propicio para su difusión. Justamente, una revista que plantea a los estudios literarios y discursivos como su campo de interés, se ofrece al mismo tiempo como zona de contacto e intercambio entre modos diversos pero muchas veces complementarios de acceder al mismo archivo, en una convergencia propiciada al mismo tiempo por la ampliación de la mirada de los estudios literarios generada por el giro material en la investigación, comentado por Hernán Pas en este mismo número.

En el número 13 de la revista (2009) aparece el trabajo de Mara Glozman, "La Academia Argentina de Letras y el peronismo (1946-1956)", riguroso examen de un corpus amplio de materiales que forman parte de la imprescindible investigación de la autora en su tesis doctoral (dirigida por Elvira Arnoux), consolidada en el valioso volumen publicado en 2015 bajo el título de Lengua y peronismo. Glozman, que hace del trabajo y la reflexión sobre el archivo ejes fundamentales de su investigación, indagaba en este caso en un profuso volumen de materiales, que comprendían no solamente las publicaciones de la Academia, sino también los discursos presidenciales, los planes de gobiernos, diarios de sesiones, las representaciones sobre la lengua y su relación con las proyecciones desde el Estado sobre la concepción de una identidad lingüístico cultural de la nación (Glozman, 142). Lengua y Estado son objeto de análisis una vez más, también desde el enfoque de la glotopolítica, en el caso de la contribución de Malena Kornfeld (Vol. 23/2, 2019), "Notas sobre la neutralidad lingüística en los medios: los Manuales de estilo de Educ.ar (2012-2015)", esta vez sobre un corpus más reciente referido a la regulación política de las prácticas lingüísticas en los medios de comunicación, más específicamente observando las estrategias asumidas por los Manuales de Estilo de los canales de televisión dependientes del Ministerio de Educación argentino, Encuentro y Pakapaka, entre 2012 y 2015. Kornfeld, especialista en lingüística descriptiva que sin embargo había realizado valiosos aportes a este campo de investigación (como el trabajo citado aquí como precedente, "Políticas de la neutralidad", en el tomo II del Homenaje a Elvira 
Arnoux publicado en 2017 por la Facultad de Filosofía y Letras de la Universidad de Buenos Aires, o la compilación De lenguas y patrias, que contaba con un muy interesante capítulo a cargo de Mara Glozman, en línea con el trabajo comentado más arriba), realiza un recorrido por las opciones adoptadas en este terreno por los medios mencionados, para desarrollar una política lingüística propia, dando forma a "un modelo de lengua alternativo al que la élite dirigente estableció a comienzos del XX a partir de una actitud conservacionista hacia la lengua y que se filtró en las concepciones casticistas, puristas y normativas sobre la lengua que aún hoy impregnan la educación argentina” (Kornfeld 53). $\mathrm{Al}$ mismo tiempo, más allá de la tradición normativa arraigada en la educación local, este modelo alternativo de la variedad de referencia para estos medios públicos argentinos se distancia a la vez de la normativa panhispánica ordenada en Madrid y del modelo de neutralidad lingüística dominante en el mercado latinoamericano de los medios de comunicación.

También desde el marco de la glotopolítica latinoamericana, y en la línea de una investigación doctoral asimismo dirigida por Arnoux, es que Florencia Rizzo examina en su artículo publicado en el número 3 del volumen 20 (2016) la configuración de la matriz discursiva de los Congresos Internacionales de la Lengua Española, cuyo interés estratégico para el área había quedado establecido en el ensayo de cierre de la fundamental Battle over Spanish de Gabriel-Stheeman y Del Valle ("Codo con codo"), y la autora ha examinado en profundidad en una amplia investigación, de la que aquí se presenta la sección correspondiente al antecedente fundacional a finales del XIX del espectáculo de la lengua que el Estado español y las empresas estratégicas del país desplegadas por el continente orquestan desde finales del siglo XX hasta el día de hoy en diversas ciudades de España y sus antiguas colonias.

De este modo, desde 2009 hasta el presente, Anclajes se ha constituido en uno de los espacios de referencia para el conocimiento de un campo en sostenida expansión y articulación con otros de más larga tradición como espacios definidos y consolidados de investigación. Una combinación de creciente prestigio y hospitalaria vocación de apertura han hecho de Anclajes un espacio no solamente de referencia, sino también de eventual construcción de diálogos críticos, que exceden naturalmente las páginas de la publicación pero encuentran no obstante en ellas una continuidad propia. Tal es el caso en lo que respecta al eje de lengua y propiedad en la construcción de los estados americanos a través de la incorporación del discurso de las modernas ciencias del lenguaje en el de los nacionalismos criollos. Este hilo se inicia con el aporte de Hernán Pas en el vol. 16, n. 2 (2012) "¿Ecos de Lautaro?: las lenguas indígenas como patrimonio cultural del nacionalismo criollo en el siglo XIX”, y puede seguirse en la intervención de quien firma esta nota en el vol. 18, n. ${ }^{\circ} 2$ (2014) "El uso, la propiedad y el valor en el debate de la lengua americana”, el trabajo de María Carolina Domínguez "Un diálogo con la tradición clásica latina en la Argentina del siglo XIX: continuidades y rupturas" (vol. 20, n. ${ }^{\circ} 3,2016$ ), el aporte de Esteban Lidgett "La 
propiedad de la lengua: norma y uso en la obra de Ricardo Monner Sans" (vol. 22, n. $\left.{ }^{\circ} 1,2018\right)$, y finalmente el más reciente aporte de Sofía de Mauro, "La imposibilidad moral de la existencia del drama: notas sobre la controversia del Ollantay" (vol. 24, n. ${ }^{\circ} 1,2020$ ). Se trata de estudios sobre recortes de diversa extensión y especificidad en el corpus de la historiografía lingüística, filológica y literaria americana, donde sin embargo se encuentra un punto fuerte de convergencia tanto en el planteo explícito del problema teórico, histórico y político de la propiedad de la lengua en sus variantes (purismo, patrimonialización), y los modos de relacionarse la intelectualidad americana con la tradición europea a la hora de pensar una matriz teórica e ideológica para la gestión política de las prácticas lingüísticas en la región.

El giro archivístico, la atención a la materialidad de los procesos culturales, ha traído consigo un renovado interés en el estudio del material epistolar, más allá del anecdotario anexo a la biografía de los grandes autores, como corpus de interés para distintas disciplinas, entre las que cuenta por supuesto la historiografía lingüística, tal como puede constatarse en la proliferación de publicaciones recientes sobre la materia, como el dossier sobre Intercambios epistolares entre lingüistas y flólogos en la primera mitad del siglo XX a cargo de Graciela Salto y Gloria Chicote en la Revista Internacional de Lingüistica Iberoamericana. Es una de las editoras de este dossier, Gloria Chicote, quien realiza en el número 1 del vol. 24 de Anclajes (2020) un aporte destacable en este sentido con "Intercambio epistolar entre Ricardo Rojas y Ramón Menéndez Pidal: fragmentos para la construcción de una hispanidad post-imperial". Este trabajo reviste un especial valor para el estudio de la historia de la consolidación de los estudios filológicos y lingüísticos en el país, ya que, como se sabe, es a través del contacto entre Rojas y Menéndez Pidal que se va a organizar entre 1922 y 1923 el Instituto de Filología de la Universidad de Buenos Aires, vital para la consolidación de un campo científico para los estudios de la lengua en el país (Toscano y García "Materiales").

La dimensión glotopolítica de la obra de Rojas, por otra parte, había sido ya objeto de un artículo publicado en el volumen 22, número 2 (2018) por Carolina Bartalini, "Del otro al yo: ideologías lingüísticas y nacionalismo cultural en Archipiélago de Ricardo Rojas", quien expande el análisis glotopolítico iniciado por Diego Bentivegna sobre este libro tan rico como escasamente estudiado, al menos desde esta perspectiva. Así, el recorrido de la autora por las representaciones ideológicas sobre el lenguaje presentes en el texto de Rojas, pone en evidencia un desplazamiento notable en una de las figuras centrales del imaginario del Centenario con respecto al deber-ser glotopolítico del Estado-nación argentino, y así concluye: "Archipiélago es un documento sobre la muerte de una lengua y el exterminio de una cultura, pero también hay aquí un sujeto que escribe revisando su esquema de percepción, una retrospectiva en tiempo presente, un espacio marginal a la formación estatal, nacional e, incluso, biográfica" (Bartalini 17). Este trabajo, por otra parte, puede pensarse asimismo integrado al diálogo 
planteado más arriba a partir de los “¿Ecos de Lautaro?” de Pas y los trabajos de De Mauro, Domínguez, Ennis y Lidgett.

Finalmente, en este mismo número encontramos un aporte de un autor al que ya se ha mencionado en estas líneas: Guillermo Toscano y García. Desde el enfoque de la historiografía lingüística y en la línea trabajada por su equipo en los últimos ańos acerca de la gramática escolar, este autor incorpora una modalidad del archivo de reciente y creciente interés para diversas formas de la investigación histórica (ese "pasado audible" que interrogara J. Sterne, en general, y en particular el fenómeno de la radio en la Argentina, tal como ha sido estudiado, por ejemplo, por Andrea Matallana, Robert Claxton o Matthew Karush), y presente ya en algunos estudios relativos a las políticas, debates y discursos sobre la lengua en la Argentina del siglo XX (Vitale, Marsimián, Ennis y Sesnich): la radiofonía. "Ondas que llevan idioma nacional. Las Lecciones de Florencio Garrigós en la Escuela del Aire" da cuenta de un corpus poco o nada conocido de intervenciones radiales a cargo de quien fuera el responsable de las "Gramaticales y filológicas" de La Prensa por largos años, además de contribuir en esta línea con publicaciones de la popularidad de Caras y caretas y El hogar. En el marco de la Escuela del Aire, "una iniciativa pionera en la Argentina en el campo de la educación a distancia mediante el uso de la radiofonía", las Lecciones de idioma nacional de Garrigós constituyen la primera lección brindada a través de ese medio, confirmando la vigencia de la importancia de la cuestión del idioma en la concepción de la formación de la ciudadanía desde el Estado a través del sistema educativo. Estas lecciones constituyeron una propuesta novedosa tanto en la forma técnica de su difusión como en las decisiones tomadas en cuanto a su contenido, donde se proponía una forma de la norma culta más próxima a las características del habla local (al menos las fonéticas y de extensión generalizada) y no a la norma culta peninsular aún promovida por el discurso académico y los instrumentos lingüísticos al uso.

El cuarto de siglo que Anclajes lleva presente en el horizonte de nuestra producción intelectual aparece, francamente, como una ocasión de celebración, naturalmente, pero de una celebración atravesada además por un sentimiento de gratitud. Lo que se agradece en este caso es la empresa sostenida, con rigor y empecinada dedicación, de ensanchar el campo de nuestro trabajo, de sostener un horizonte amplio y hospitalario sobre el suelo firme de un trabajo bien hecho, de manera ejemplar. Pienso en la palabra ejemplar en este caso, porque justamente lo que ha sabido ofrecer es un modelo de trabajo y un espacio de referencia. No solo con Anclajes, porque el despliegue del trabajo individual y colectivo tiene muchas otras vertientes, pero especialmente a través de su plataforma, el Instituto de Investigaciones Literarias y Discursivas de la Universidad Nacional de La Pampa logró algo fundamental para cualquier centro de investigación, y es que se quiera, necesite, no se pueda evitar hablar con quienes lo integran. Y lograr eso, desde una institución situada en las puertas de la Patagonia, prácticamente en el centro geográfico pero lejos de los centros económico-políticos 
del país, es ya una gran cosa. Pero lo más interesante del asunto es que no se trata solamente de ganar visibilidad y protagonismo para un espacio y sus actores, sino, mucho más importante aún, de hacer de este un espacio de cruces, intercambios, diálogos, logrando algo en extremo complejo como la convivencia entre el rigor y la hospitalidad de sus páginas, el cuidado y la puntualidad de su edición. Porque cualquiera que haya editado una revista científica, al menos en el amplio campo que se conoce a grandes rasgos como el de las letras, sabe de las dificultades de mantener la periodicidad, sobre todo si se tiene la generosa osadía de que esta sea cuatrimestral, como sucede con Anclajes desde hace al menos un lustro. Al mismo tiempo, cualquiera que haya intentado sostener una revista en universidades, nuevamente, distantes de los centros más populosos y de largas trayectorias en la construcción científica, sabe que hay que hacer de la necesidad virtud, y concebir una amplitud temática y disciplinar que sostenga a la vez una identidad. Y eso, en Anclajes, de un modo notable, sucede. Y por eso también para quienes transitamos el espacio necesariamente amplio, poroso, muchas veces interdisciplinario del estudio de los discursos legos o especializados sobre el lenguaje, aquellos que contribuyen a la construcción discursiva de las lenguas como artefactos políticos, Anclajes se ha convertido también, necesariamente, en lo que es para otras líneas de trabajo en el área: un punto de referencia, un polo de atracción, un espacio para el diálogo.

\section{Referencias bibliográficas}

Arnoux, Elvira Narvaja de. "La Glotopolítica: transformaciones de un campo disciplinario", Lenguajes: teorías y prácticas. Buenos Aires, Gobierno de la Ciudad de Buenos Aires, Secretaría de Educación, 2000, pp. 95-109.

Arnoux, Elvira Narvaja de. Los discursos sobre la nación y el lenguaje en la formación del Estado (Chile, 1842-1862). Estudio glotopolítico. Buenos Aires, Santiago Arcos, 2008.

Arnoux, Elvira Narvaja de y José del Valle. "Las representaciones ideológicas del lenguaje. Discurso glotopolítico y panhispanismo", dossier Ideologías lingüisticas y el español en contexto histórico; Spanish in Context vol. 7, n. ${ }^{\circ}$ 1, 2010, pp. 1-24, https://doi.org/10.1075/sic.7.1.01nar.

Arnoux, Elvira Narvaja de, José del Valle y Alexandre Duchêne. "Glottopolitique - glotopolítica: circulation, appropriation et expansion d'une lecture sociale du langage". Glottopol. Revue de sociolinguistique en ligne, 32, 2019, pp. 2-6.

Bartalini, Carolina. "Del otro al yo: ideologías lingüísticas y nacionalismo cultural en Archipiélago de Ricardo Rojas". Anclajes, vol. 22, n. ${ }^{\circ}$ 2, 2018, pp. 1-19, https://doi.org/10.19137/anclajes-2018-2221. 
Bauman, Richard y Charles L. Briggs. Voices of Modernity. Language Ideologies and the Politics of Inequality. Cambridge, Cambridge University Press, 2003.

Benes, Tuska. In Babel's shadow. Language, Philology and the Nation in Nineteenth Century Germany. Detroit, Wayne State University Press, 2008.

Bentivegna, Diego. "Ricardo Rojas y Benvenuto Terracini: destierro, muerte y biopolítica de las lenguas". Filología, vol. 46, 2014, pp.125-138.

Blommaert, Jan (ed.). Language Ideological Debates, Berlín/Nueva York, Mouton de Gruyter, 1999.

Chicote, Gloria. "Intercambio epistolar entre Ricardo Rojas y Ramón Menéndez Pidal: fragmentos para la construcción de una hispanidad post-imperial". Anclajes, vol. 24, n. ${ }^{\circ}$ 1, 2020, pp. 1-18, https://doi.org/10.19137/anclajes2020-2411.

Claxton, Robert. From Parsifal to Perón: Early Radio in Argentina, 1920-1944. Gainesville, University Press of Florida, 2007.

Crowley, Tony. Language in History. Londres/Nueva York, Routledge, 1986.

De Mauro, Sofía. "La imposibilidad moral de la existencia del drama: notas sobre la controversia del Ollantay". Anclajes, vol. 24, n. ${ }^{\circ}$ 1, 2020, pp. 69-86, https://doi.org/10.19137/anclajes-2020-2415.

Del Valle, José. La lengua, ¿patria común? Ideas e ideologías del español. Madrid/ Fráncfort del Meno, Vervuert/Iberoamericana, 2005.

Del Valle, José (ed.). A Political History of Spanish. The Making of a Language. Cambridge (e.o.), Cambridge University Press, 2013.

Del Valle, José (ed.). Historia politica del español. La invención de una lengua. Madrid, Aluvión, 2015.

Del Valle, José y Luis Gabriel-Stheeman. "'Codo con codo'. Hispanic community and the language spectacle", The Battle over Spanish between 1800 and 2000. Language ideologies and Hispanic intellectuals, Londres/Nueva York, Routledge, 2002, pp. 193-216.

Del Valle, José, Daniela Lauria, Mariela Oroño y Darío Rojas (eds.). Autorretrato de un idioma. Crestomatía glotopolítica del español. Madrid, Lengua de trapo, 2021.

Domínguez, María Carolina. "Un diálogo con la tradición clásica latina en la Argentina del siglo XIX: continuidades y rupturas". Anclajes vol. 20, n. ${ }^{\circ} 2$, 2016, pp. 17-32, https://doi.org/10.19137/anclajes-2016-2022.

Ennis, Juan. "El uso, la propiedad y el valor en el debate de la lengua americana". Anclajes, vol. 18, n. ${ }^{\circ}$ 2, 2014, pp. 32-47.

Ennis, Juan y Laura Sesnich. "Print (and Broadcasting) Capitalism in Times of Crisis: The Quest for a Unified Language Market in Buenos Aires, 1935- 
1943”. Bulletin of Spanish Studies, vol. 97, n. ${ }^{\circ}$ 7, 2020, pp. 1211-1233, https://doi.org/10.1080/14753820.2020.1815978.

Foucault, Michel. Les Mots et les choses. Une archéologie des sciences humaines. París, Gallimard, 1967.

Glozman, Mara. "La Academia Argentina de Letras y el peronismo (19461956)". Anclajes, vol. 13, n. ${ }^{\circ} 13,2009$, pp. 129-144.

Glozman, Mara. Lengua y peronismo. Políticas y saberes lingüisticos en la Argentina, 1943-1956. Buenos Aires, Biblioteca Nacional, 2015.

Guespin, Louis y Jean-Baptiste. Marcellesi. "Pour la Glottopolitique". Langages, n. 83,1986 , pp. 5-34, https://doi.org/10.3406/lgge.1986.2493.

Harris, Roy. The Language Myth. Londres, Duckworth, 1981.

Heller, Monica y Bonnie McElhinny. Language, Capitalism, Colonialism: Towards a Critical History. Toronto, University of Toronto Press, 2017.

Karush, Matthew. Cultura de clase. Radio y cine en la creación de una Argentina dividida (1920-1946). Buenos Aires, Ariel, 2013.

Kornfeld, Laura Malena (comp.). De lenguas, ficciones y patrias. Los Polvorines, Universidad Nacional de General Sarmiento, 2014.

Kornfeld, Laura Malena. "Políticas de la neutralidad", Homenaje a Elvira Arnoux. Estudios de análisis del discurso, glotopolítica y pedagogía de la lectura y la escritura, editado por Roberto Bein et al., Tomo II: Glotopolítica. Buenos Aires, Editorial de la Facultad de Filosofía y Letras, 2017, pp. 67-81.

Kornfeld, Laura Malena. "Notas sobre la neutralidad lingüística en los medios: los Manuales de estilo de Educ.ar (2012-2015)". Anclajes vol. 23, n. ${ }^{\circ} 2$, 2019, pp. 39-55, https://doi.org/10.19137/anclajes-2019-2323.

Lidgett, Esteban. "La propiedad de la lengua: norma y uso en la obra de Ricardo Monner Sans". Anclajes, vol. XXII, n. ${ }^{\circ} 1,2018$, pp. 55-71, https://doi.org/ 10.19137/anclajes-2018-2214.

Marsimián, Silvina. Lengua, radio, humor y censura: el caso Nini Marshall. Buenos Aires, Biblos, 2016.

Matallana, Andrea. Locos por la radio: una historia social de la radiofonía argentina, 1923-1947. Buenos Aires, Prometeo, 2006.

Pas, Hernán. “¿Ecos de Lautaro?: las lenguas indígenas como patrimonio cultural del nacionalismo criollo en el siglo XIX”. Anclajes, vol. XVI, n. ${ }^{\circ} 2,2012$, pp. 74-92.

Rizzo, María Florencia. "Los congresos de la lengua española: configuración de una matriz discursiva". Anclajes, vol. 20, n. ${ }^{\circ} 3,2016$, pp. 59-75, https://doi. org/10.19137/anclajes-2016-2034 
Sánchez-Macedo, Jaime. "El giro archivístico; su impacto en la investigación histórica". Humanitas. Anuario del Centro de Estudios Humanísiticos, vol. IV, n. ${ }^{\circ} 47,2020$, pp. 183-223.

Sterne, Jonathan. The Audible Past. Cultural Origins of Sound Reproduction. Durham /Londres, Durham University Press, 2003.

Tello, Andrés Maximiliano. Anarchivismo. Tecnologías políticas del archivo. Adrogué, La Cebra, 2018.

Toscano y García, Guillermo. "Materiales para una historia del Instituto de Filología de la Universidad de Buenos Aires (1920-1926)". Revista Internacional de Lingüistica Iberoamericana, vol. VII, 1, n. ${ }^{\circ}$ 13, 2009, pp. 113-135.

Toscano y García, Guillermo. "Ondas que llevan idioma nacional. Las Lecciones de Florencio Garrigós en la Escuela del Aire”. Anclajes, vol. 25, n. ${ }^{\circ} 3,2021$, pp. 117-135, https://doi.org/10.19137/anclajes-2021-25314.

Vitale, María Alejandra. "En torno a la lengua en la radio argentina: la encuesta de la Comisión Parlamentaria de 1938". Elvira Arnoux y Roberto Bein (eds.). La regulación política de las prácticas lingüísticas. Buenos Aires, Eudeba, 2010, pp. 73-99.

Williams, Raymond. Marxism and Literature. Oxford, Oxford University Press, 1977.

Woolard, Kathryn y Schieffelin, Bambi. "Language ideology". Annual Review of Anthropology, vol. 23, 1994, pp. 55-82, https://doi.org/10.1146/annurev. an.23.100194.000415. 